\title{
Restrictive Cardiomyopathy With Right-Sided Dominant Heart Failure After Sinus Conversion From Atrial Fibrillation Case Report
}

\author{
Noriko Sasaki, MD; Yoshio Yasumura, MD; Nobuyuki Uemura, MD; \\ Akihisa Hanatani, MD; Satoshi Nakatani, MD; \\ Masakazu Yamagishi, MD; Kunio Miyatake, MD
}

\begin{abstract}
A 44-year-old woman with restrictive cardiomyopathy showed right-sided dominant heart failure after conversion to sinus rhythm from paroxysmal atrial fibrillation (AF). During cardiac catheterization, the hemodynamics were compared before and after cardioversion; that is, with AF and with sinus rhythm. The hemodynamic parameters worsened after conversion to sinus rhythm, with a significant decrease in heart rate, but improved with atrial pacing. Pacemaker implantation was performed to avoid bradycardia while keeping the sinus rhythm with amiodarone. As a result, the patient has been free from symptoms of heart failure for almost 1 year and her serum brain natriuretic peptide level also improved remarkably. (Circ J 2003; 67: 969-971)
\end{abstract}

Key Words: Atrial fibrillation; Cardioversion; Restrictive cardiomyopathy

$\mathbf{T}$ There have been previous cases of left-sided heart failure soon after successful electrical cardioversion,-8 but the mechanism of this curious phenomenon has not yet been clarified completely. One possible cause is that left atrial mechanical activity recovers much more slowly than that of the right atrium! The underlying cardiovascular disorders of these patients were rheumatic heart disease, hypertrophic cardiomyopathy, ischemic heart disease, and congenital heart disease. We present an intriguing case of restrictive cardiomyopathy that developed right-sided dominant heart failure whenever atrial fibrillation (AF) was converted to sinus rhythm spontaneously or by electrical cardioversion. We report the interesting hemodynamic profile during both $\mathrm{AF}$ and sinus rhythm that suggests the possible mechanism of right-sided heart failure, and the therapy that has successfully controlled recurrence of heart failure in this patient.

\section{Case Report}

A 44-year-old woman was referred to hospital in March, 2000 complaining of palpitation, heaviness in the right upper quadrant and dyspnea on exertion after a palpitation. She had had some cardiac disease diagnosed by her home doctor at 16 years of age, and had experienced occasional palpitations thereafter. At 38 years old, she sometimes felt a heaviness in the right upper quadrant, and had dyspnea when she tried to continue her work in this condition. At 40 years old, the frequency of the palpitation attacks increased

(Received December 6, 2001; revised manuscript received May 7, 2002; accepted May 10, 2002)

Cardiology Division of Medicine, National Cardiovascular Center, Suita, Japan

Mailing address: Noriko Sasaki, MD, Cardiology Division of Medicine, National Cardiovascular Center, 5-7-1 Fujishiro-dai, Suita 5658565, Japan and she was diagnosed as paroxysmal AF the next year. Medication did not control the symptoms. When the AF was converted to sinus rhythm, the feeling of heaviness in the right upper quadrant developed, with a significant increase in hepatic enzymes. In contrast, these symptoms improved during AF. The patient had also had hypothyroidism since the age of 40 years, but had become euthyroid without medication. Her mother also had had AF since the age of 40 years and after one episode of cardiac failure, she was diagnosed as cardiomyopathy.

On admission, the present patient's height was $147 \mathrm{~cm}$, and her weight was $53 \mathrm{~kg}$. Her blood pressure was $106 / 66 \mathrm{mmHg}$ and the pulse rate was 125 beats/min and irregular. The third heart sound was audible. The liver was palpable with tenderness 2 fingerbreadths below the xiphoid process. There was no apparent edema and her palpebral conjunctivae were not anemic.

Chest X-ray revealed a cardiothoracic ratio of $60 \%$ and mild lung congestion (Fig 1). The ECG showed atrial flutter-fibrillation at a heart rate 143 beats/min with incomplete right bundle branch block and evident left ventricular hypertrophy (Fig 1).

Laboratory tests revealed elevated hepatic enzymes (aspartate aminotransferase 59 U/L, alanine aminotransferase $79 \mathrm{U} / \mathrm{L}, \mathrm{Y}$-glutamyl transpeptidase $79 \mathrm{U} / \mathrm{L}$ ), possibly because of liver congestion, and serum atrial natriuretic peptide (ANP) $(200 \mathrm{pg} / \mathrm{ml})$ and brain natriuretic peptide (BNP) $(657 \mathrm{pg} / \mathrm{ml})$ concentrations were also increased. Serum thyroid stimulating hormone (TSH) was mildly elevated (freeT3 $3.0 \mathrm{pg} / \mathrm{ml}$, freeT $41.1 \mathrm{ng} / \mathrm{dl}$, TSH $12 \mu \mathrm{IU} / \mathrm{ml}$ ) and anti-thyroid antibodies were positive (anti-thyroglobulin antibody $\times 400$, anti-microsome antibody $\times 25,600$ ) possibly from the chronic thyroiditis.

An echocardiogram showed mild left ventricular hypertrophy and diffuse mild reduction of the left ventricular wall motion; the thickness of the interventricular septum 

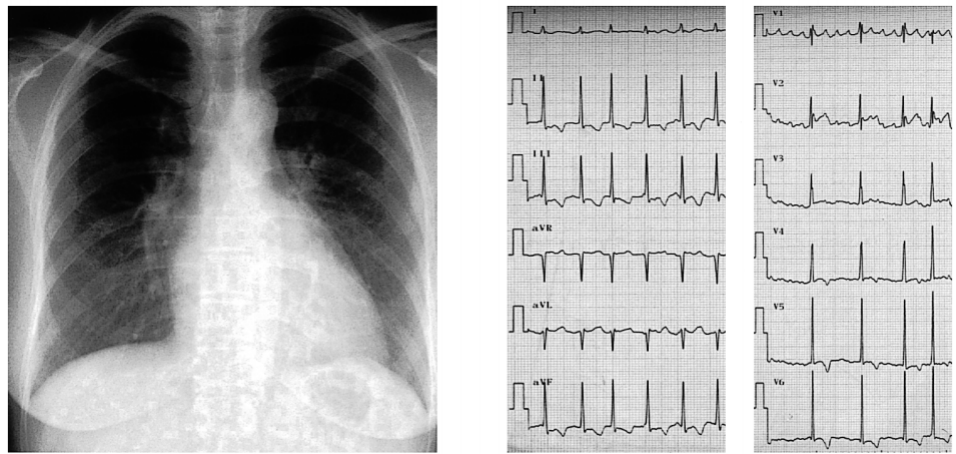

Fig 1. Chest X-ray shows a cardiothoracic ratio of $60 \%$ and mild lung congestion (Left). Twelve-lead ECG shows atrial flutter-fibrillation during a heart rate of 143 beats/min with incomplete right bundle branch block.

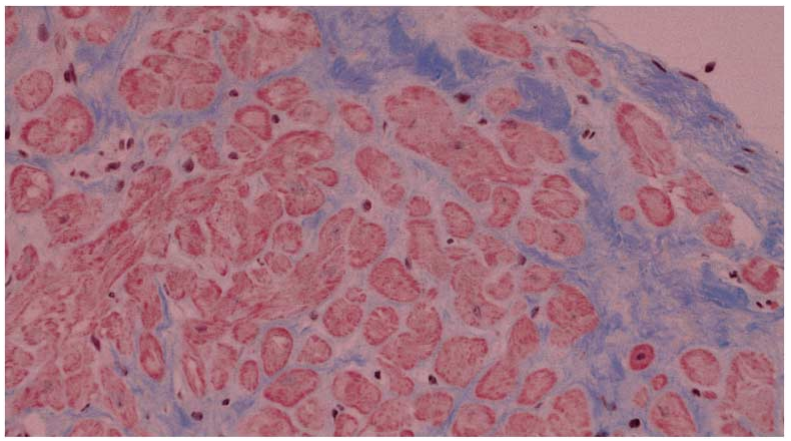

Fig 2. Histopathology of the myocardial biopsy from the right side of the interventricular septum. There is moderate fibrosis but no sign of amyloidosis or sarcoidosis (Masson's trichrome stain).

Table 1 Hemodynamic Changes Before and After Electrical Cardioversion

\begin{tabular}{lcc}
\hline \hline & $A f$ & $S R$ \\
\hline$H R($ beats/min $)$ & 109 & 52 \\
$P C W P(\mathrm{mmHg})$ & 17 & 22 \\
$P A P(\mathrm{mmHg})$ & $32 / 15(23)$ & $38 / 20(27)$ \\
$R V P(\mathrm{mmHg})$ & $32 / \sim 8$ & $37 / \sim 16$ \\
$R A P(\mathrm{mmHg})$ & 9 & 13 \\
$L V P(\mathrm{mmHg})$ & $104 / \sim 14$ & $94 / \sim 16$ \\
$C O($ L $/ \mathrm{min})$ & 3.5 & 2.9 \\
\hline
\end{tabular}

Af, atrial fibrillation; SR, sinus rhythm; HR, heart rate; $P C W P$, pulmonary capillary wedge pressure; PAP, pulmonary artery pressure; $R V P$, right ventricular pressure; RAP, right atrial pressure; LVP, left ventricular pressure; $\mathrm{CO}$, cardiac output.

(IVS) was $11 \mathrm{~mm}$, posterior wall thickness (PW) $12 \mathrm{~mm}$, end-diastolic left ventricular diameter (LVDd) $41 \mathrm{~mm}$, end-systolic left ventricular diameter (LVDs) $30 \mathrm{~mm}$, and fractional shortening (FS) 26\%. Pulsed wave Doppler transmitral flow velocities showed a restrictive pattern during sinus rhythm.

On cardiac catheterization, coronary angiography showed no significant stenosis. The left ventricular enddiastolic volume index determined by left ventriculography was $49 \mathrm{ml} / \mathrm{m}^{2}$, and the ejection fraction was $61 \%$. The pressure pattern was dip-and-plateau in both ventricles. The right atrial pressure was mildly elevated $(9 \mathrm{mmHg})$ and mild pulmonary hypertension (mean pulmonary arterial pressure, $23 \mathrm{mmHg}$ ) was observed during AF. During right heart catheterization, small biopsies were taken from the IVS and these showed severe fibrosis, but neither amyloidosis nor sarcoidosis (Fig 2). We diagnosed restrictive cardiomyopathy because of the unremarkable left ventricular
Table 2 Hemodynamic Changes With Increased Heart Rate During Atrial Pacing

\begin{tabular}{ccccc}
\hline \hline $\begin{array}{l}H R \\
\text { (beats/min) }\end{array}$ & $\begin{array}{c}C O \\
(\text { L/min })\end{array}$ & $\begin{array}{c}P A P \\
(\mathrm{mmHg})\end{array}$ & $\begin{array}{c}R A P \\
(\mathrm{mmHg})\end{array}$ & $\begin{array}{c}R V P \\
(\mathrm{mmHg})\end{array}$ \\
\hline 50 & 3.58 & $42 / 15(25)$ & 8 & $42 / \sim 13$ \\
70 & 4.66 & $39 / 11(22)$ & $*$ & $*$ \\
90 & 5.08 & $34 / 10(17)$ & 4 & $35 / \sim 4$ \\
110 & $*$ & $32 / 9(16)$ & $*$ & $*$
\end{tabular}

HR, heart rate; PAP, pulmonary artery pressure; RVP, right ventricular pressure; RAP, right atrial pressure; $C O$, cardiac output; *, not done.

Table 3 Changes in Echocardiographic Parameters With Increased Heart Rate During Atrial Pacing

\begin{tabular}{lcccc}
\hline \hline $\begin{array}{l}\text { HR } \\
\text { (beats/min })\end{array}$ & $\begin{array}{c}\text { CO } \\
(\text { L/min })\end{array}$ & $\begin{array}{c}S V \\
(\mathrm{ml})\end{array}$ & E/A & $\begin{array}{c}\text { DcT } \\
(\mathrm{ms})\end{array}$ \\
\hline 50 & 3.20 & 64 & $0.57 / 0.41$ & 123 \\
70 & 3.99 & 57 & $0.45 / 0.49$ & 220 \\
80 & $*$ & $*$ & $0.39 / 0.57$ & 175 \\
90 & 3.87 & 43 & $0.39 / 0.73$ & summation \\
\hline
\end{tabular}

$H R$, heart rate; $C O$, cardiac output; SV, systolic volume; DcT, deceleration time; *, not done.

dilatation, normal ejection fraction, bilateral dip-andplateau pattern and the endocardial histology.

During diagnostic cardiac catheterization, we compared the hemodynamics before and after cardioversion; that is, during AF and in sinus rhythm. The heart rate during sinus rhythm was approximately 50 beats/min and the hemodynamics were worse than during $\mathrm{AF}$ (Table 1). The deterioration in the hemodynamics suggested a reduction in cardiac output and subsequent rise in right atrial pressure (RAP), pulmonary capillary wedge pressure (PCWP), and both left and right end-diastolic ventricular pressures (LVEDP, RVEDP). We investigated the effect of heart rate on cardiac function by atrial pacing. The more the heart rate increased, the more the hemodynamics improved (Table 2).

The patient had 2 types of symptom: palpitation during $\mathrm{AF}$ when the heart rate increased to approximately 150 beats/min on exertion, as well the feeling of heaviness in the right upper quadrant with dyspnea on exertion after spontaneous recovery of sinus rhythm. The controlling the heart rate during $\mathrm{AF}$ has been difficult despite using various drugs, such as digitalis, class Ia drugs, calcium antagonists, and $\beta$-blockers. Therefore, we selected to maintain sinus rhythm and control the heart rate by an implanted pacemaker. While maintaining sinus rhythm with $200 \mathrm{mg} /$ day of amiodarone after electrical cardioversion, a pacemaker 
was implanted (AAIR, rate 70 beats/min) to prevent sinus bradycardia. Pulsed Doppler echocardiographic evaluation of the transmitral flow velocitiy showed an alteration from the restrictive pattern to an abnormal relaxation pattern accompanied with an increase in heart rate from 50 beats $/ \mathrm{min}$ to 80 beats/min (Table 3). A week after pacemaker implantation, the cardiothoracic ratio had decreased from $54 \%$ to $48 \%$, the enlarged liver had reduced in size, and both the serum ANP and BNP concentrations had also decreased (120 to $72 \mathrm{pg} / \mathrm{ml}$ and 244 to $160 \mathrm{pg} / \mathrm{ml}$, respectively). The patient has been free from both symptoms and signs of heart failure since then and her New York Heart Association functional class has been class I for at least 1 year.

\section{Discussion}

Left-sided heart failure, namely pulmonary edema, can occur after electrical cardioversion of $\mathrm{AF}$ to sinus rhythm! ${ }^{1-6,8}$ Upshaw reported the hemodynamic aspects and possible causes of this phenomenon; he observed that cardiac output decreased after cardioversion in more than one-third of the patients, and it took more than 4 weeks to gradually increase! One of the causes of this delayed recovery is considered to be the gradual recovery of atrial function, in particular, delayed recovery of left atrial function compared with that of the right. Pulmonary and/or coronary artery emboli and the myocardial damage by direct current countershock may be additional causative factors of the pulmonary edema. Although the present patient also developed heart failure after either cardioversion or spontaneous recovery to sinus rhythm, she is unique in the development of right-sided dominant heart failure.

Why did the right-sided heart failure develop after cardioversion of AF to sinus rhythm? We compared the hemodynamics during $\mathrm{AF}$ and sinus rhythm and found that the heart rate decreased after conversion to sinus rhythm with elevated mean RAP, PCWP and LVEDP, and reduced cardiac output (Table 1). The hemodynamics improved with increases in the heart rate during right atrial pacing in sinus rhythm (Table 2). Therefore, in this patient the heart rate must play an important role in the difference in the hemodynamics during $\mathrm{AF}$ and sinus rhythm. A heart rate less than 50 beats/min may increase the end-diastolic volume of both ventricles and in this case increased stiffness because of the restrictive cardiomyopathy may have caused the remarkable increase in end-diastolic pressure accompanied by a small increase in end-diastolic volume. Indeed, the PCWP was $22 \mathrm{mmHg}$ and RAP $13 \mathrm{mmHg}$ during sinus rhythm with a heart rate of 52 beats $/ \mathrm{min}$. As is often the case with biventricular dysfunction, right-sided heart failure may have masked apparent left-sided heart failure.

The patient's heart rate could not be controlled medically during $\mathrm{AF}$ and it increased remarkably with symptoms on exertion. In addition, during sinus rhythm the heart rate was low enough to cause right-sided heart failure. Bradycardia assists diastolic filling time in patients with diastolic dysfunction, but excessive bradycardia can decrease cardiac performance and the force-frequency relationship. Therefore, our aim was to prevent bradycardia by atrial pacing while keeping sinus rhythm with amiodarone. We implanted a permanent pacemaker in AAI mode, and set up the pacing rate at 70 beats/min, because the deceleration time of the mitral flow velocities at that rate were the longest among the heart rates evaluated by Doppler echocardiography 6 days after electrical cardioversion (Table 3 ). It remains unknown whether the pacing rate will be always optimal when the slowness of the recovery of both the right and left atrial function is taken into account. Amiodarone was administered at $200 \mathrm{mg} /$ day initially, reducing to $150 \mathrm{mg} /$ day to maintain sinus rhythm. Subsequently, the plasma BNP concentration decreased from $244 \mathrm{pg} / \mathrm{ml}$ to $160 \mathrm{pg} / \mathrm{ml}$ and the patient has been free from symptoms of heart failure for at least 1 year.

As far as we know, this is the first report of right-sided dominant heart failure accompanying sinus conversion of $\mathrm{AF}$. Its emergence seemed to be related to a low heart rate during sinus rhythm associated with increased stiffness of the right ventricle.

\section{References}

1. Upshaw CB. Hemodynamic changes after cardioversion of chronic atrial fibrillation. Arch Intern Med 1997; 157: 1070-1076.

2. Mayosi BM, Commerford PJ. Pulmonary edema following electrical cardioversion of atrial fibrillation. Chest 1996; 109: 278-280.

3. Omran H, Jung W, Luderitz B. Dysfunction of the left atrium after cardioversion of atrial fibrillation. Am J Cardiol 1998; 82: 837.

4. Budow J, Natarajan P, Kroop IG. Pulmonary edema following direct current cardioversion for atrial arrhythmias. JAMA 1971; 218: 1803 1805.

5. Gomaa M, Dayem MK, Eissa A, Ateyya M. Pulmonary edema after direct current countershock. Chest 1972; 62: 623-625.

6. Lindsay J. Pulmonary edema following cardioversion. Am Heart $J$ 1967; 74: 434-435.

7. Scott ME, Patterson GC. Cardiac output after direct current conversion of atrial fibrillation. Br Heart J 1969; 31: 87-90.

8. Resnekov L. Haemodynamic studies before and after electrical conversion of atrial fibrillation and flutter to sinus rhythm. Br Heart $J$ 1967; 29: 700-708.

9. Navab A, La Due JS. Post conversion systemic arterial embolism. Am J Cardiol 1965; 16: 452-453.

10. Gardner JD, Dunn M. Spontaneous conversion of long-standing atrial fibrillation. Chest 1982; 81: 429-432.

11. Olsson SB, Orndahl G, Enestrom S, Eskilsson J, Persson S, Grennert $\mathrm{ML}$, et al. Spontaneous reversion from long-lasting atrial fibrillation to sinus rhythm. Acta Med Scand 1980; 207: 5-20.

12. Grim RA, Leung DY, Black IW, Stewart WJ, Thomas JD, Klein AL. Left atrial appendage 'stunning' after spontaneous conversion of atrial fibrillation demonstrated by transesophageal Doppler echocardiography. Am Heart J 1995; 130: 174-176. 